\title{
Gestión curricular holística en el modelo por competencias: un estudio exploratorio
}

\author{
Angélica Crespo Cabuto ${ }^{1}$, Sonia Verónica Mortis Lozoya ${ }^{1 *}$, y Sergio Raúl Herrera Meza² \\ (1) Departamento de Educación, Instituto Tecnológico de Sonora, 5 de febrero 818 sur, Col. Centro, Ciudad Obregón \\ Sonora. C.P. 85000, (Correo-e: angelica.crespo@itson.edu.mx; sonia.mortis@itson.edu.mx) \\ (2) Centro Universitario CIFE, Calle Tabachin 514, Bellavista. Cuernavaca Morelos, México. C.P. 62140 \\ (Correo-e: raulherreram.rh@gmail.com)
}

Recibido Dic. 4, 2020; Aceptado Feb. 3, 2021; Versión final Mar. 30, 2021, Publicado Ago. 2021

\begin{abstract}
Resumen
El propósito de este estudio exploratorio con enfoque cuantitativo, fue identificar el grado de aplicación de la gestión curricular holística en el diseño curricular en programas de pregrado en 52 universidades de nueve países de América Latina, desde la perspectiva de los gestores curriculares y docentes. Participaron 172 profesionales de la educación y se utilizó una rúbrica para valorar la metodología de la gestión curricular holística. Los resultados muestran que los programas educativos basados en el modelo curricular por competencias aplican las fases para la gestión curricular en un nivel medio bajo. Se encontraron diferencias significativas en la aplicación de algunas fases de dicha metodología entre los modelos curriculares. Se concluye que la fase de planeación del perfil y proceso de egreso es primordial debido a que en ésta se definen las competencias del perfil de egreso y deben cubrir las necesidades sociales donde se desempeñará el futuro profesionista.
\end{abstract}

Palabras clave: enfoque socioformativo; evaluación; currículo; calidad; educación; personal docente; plan; estudios universitarios

\section{Holistic curricular management in the competency and socio- formative approach: an exploratory study}

\begin{abstract}
The purpose of this exploratory quantitative-approach study was to assess the implementation degree of holistic curricular management by curricular managers and professors when designing undergraduate curricula at 52 universities from nine Latin American countries. One-hundred-and-seventy-two educators participated in this study. A rubric was used to assess the socio-formative-curriculum design methodology. The results showed that degree programs applied phases for curricular management at a low-medium level. Significant differences were found in the application of phases between degree program curricular models. It is concluded that the profiling phase and the registration process are essential for defining registration profile competences and for addressing the social needs of future professionals.
\end{abstract}

Keywords: socio-formative approach; evaluation; curriculum; quality; education; academic personnel; university study plan 


\section{INTRODUCCIÓN}

La calidad educativa es un factor fundamental en las universidades, ya que deben mantener estándares que las posicionen en el ámbito nacional e internacional, mediante criterios de evaluación o acreditación de los Programas Educativos (PE). Para lograrlo, es necesario que la evaluación curricular sea de alto impacto, ya que se espera una transformación real en el proceso educativo, que favorezca la investigación con fines de innovación, creación y atención de problemas sociales (Casarini, 2013; Parra et al, 2017; Rowe y Zegwaard, 2017).

Un PE tiene calidad cuando logra que los estudiantes aprendan a resolver problemas del entorno y trabajen para alcanzar la sociedad sostenible, aplicando los valores universales y el trabajo coordinado entre todos; además, debe tomar en cuenta las características de la sociedad del conocimiento, es por ello, que algunos ministerios o secretarias de educación han implementado el enfoque por competencias en sus diseños curriculares (Barzaga et al., 2019). Cabe agregar, que una de las principales críticas en la formación universitaria radican en la necesidad de considerar los aspectos externos de la institución para atender el contexto social, el compromiso con la formación práctica y el desarrollo de procesos formativos que revaloricen el desarrollo profesional (López et al., 2016; López et al., 2016).

Ante la situación planteada y con el objetivo de mantener la calidad educativa, las universidades llevan a cabo procesos de evaluación curricular que les permiten fundamentar la toma de decisiones para el rediseño de planes de estudio. Es decir, ejercicios participativos con el fin de obtener información sobre las fortalezas y debilidades de los elementos curriculares de los PE, con la finalidad de interpretarla (Alexander y Hjortsø, 2019; Changiz et al., 2019; Glatthorn et al., 2019). Asimismo, para lograr la calidad educativa se requieren procesos de diseño curricular reflexivos que favorezcan la articulación de los tres niveles de concreción curricular. Además, la evaluación curricular debe ser continua, sistémica, holística y reflexiva en todas sus etapas: diseño, ejecución y resultado. Lo anterior para favorecer el establecimiento de acciones de apoyo y seguimiento para el logro de las metas y mantener la pertinencia de los planes de estudio (Aguirre-Garzón, 2018; Boyle y Charles, 2016; Tobón, 2017).

Con referencia a lo anterior, la participación de los profesores en el proceso de desarrollo curricular es primordial, debido a que se requiere una transformación en los procesos de enseñanza, aprendizaje y evaluación. Es decir, para lograr un cambio en el último nivel de concreción curricular y alinear los procesos de instrucción, los profesores deben de conocer las políticas, los fines que persigue el plan de estudios, además de los elementos y procesos que conforman el currículo (García-Martínez et al., 2018; Shubert, 2018; Voogt et al., 2016).

Se han efectuado diversas investigaciones sobre el desarrollo curricular, en los cuales se han reportado resultados que contribuyen a mejorar la efectividad de este proceso. Uno de estos hallazgos, es que varias investigaciones coinciden en la importancia de contar con un equipo que lidere dicho proceso; por ejemplo, en el estudio efectuado por Changiz et al. (2019) indican que estos equipos permitirán una toma de decisiones pertinente y dar seguimiento a las fases del proceso curricular. Asimismo, los resultados de estudios efectuados por varios investigadores (Cisternas, et al. 2016; Huang, 2017; Salas, 2016), destacan la relevancia de la participación de profesores en el equipo de diseñadores curriculares, con el fin de promover una cultura de colaboración entre los gestores de programas educativos y los docentes. En otros resultados de estas investigaciones, refieren la importancia de la gestión del talento humano con el fin de motivar y empoderar a los equipos de rediseño curricular, para fomentar la reflexión de los profesores sobre su función en el proceso educativo e impulsar propuestas innovadoras (Dempster et al., 2012; Martínez et al., 2017).

Por otra parte, los resultados obtenidos por Alexander y Hjortsø (2019), Changiz et al. (2019), Icarte y Labate (2016), así como Ngassapa et al. (2012), indican que definir principios claros que guíen el proceso de diseño curricular, el cual debe ser conocido por todas las partes interesadas, favorece la comunicación y, por lo tanto, un proceso más ordenado y sistematizado. Otro aspecto esencial para lograr la calidad educativa, es que el currículo considere el abordaje de problemas reales durante el proceso de formación, ya que esto favorece el desarrollo de competencias vinculadas a las necesidades reales del contexto y la pertinencia del plan de estudios (Barzaga et al., 2019; Ceja et al., 2013; Rowe y Zegwaard, 2017). Por último, Icarte y Labate (2016), López et al., (2016), y Shubert (2018) coinciden en que la evaluación es una fase esencial del desarrollo curricular, debido a que se debe contar con mecanismos que permitan valorar el logro de las competencias, además de fungir como guía en la práctica del docente y del proceso formativo.

\section{OTROS ANTECEDENTES}

El modelo por competencias favorece el aprendizaje activo, centrado en el estudiante, donde se realiza una integración de saberes para demostrar desempeños estandarizados con fines de certificación, para dar solución a problemas del sector productivo, considerando los requerimientos de los empleadores. Es decir, a 
pesar de que este modelo surge en el campo laboral, se integra al ámbito educativo como una propuesta muy pertinente para vincular a la educación superior con las organizaciones, debido a que considera las necesidades del entorno laboral y social (Casanova, et al., 2018; Díaz, Poblete y Gallardo, 2019).

Con relación al modelo conductista, ha sido utilizado desde el siglo pasado, a partir de los estudios de J. B. Watson (1879-1958) quien es considerado como el fundador del conductismo debido a sus aportaciones en: "la publicación de su principal contribución: "Psychology as the Behaviorist Views it", conocida como "el manifiesto conductista" y publicada en el año 1913 (Pellón, 2013, p. 390). Este modelo se caracteriza por favorecer la certificación individual, las cuales se alcanzan al demostrar comportamientos estandarizados, los cuales son necesarios para cubrir las demandas laborales; es decir, el aprendizaje se mide mediante conductas observables, que se refuerzan con estímulos que propician respuestas positivas (Pellón, 2013; Posso et al., 2020)

Por otra parte, desde el modelo constructivista se busca una integración y transformación desde el aprendizaje significativo a través de la aplicación de lo aprendido en la resolución de problemas cotidianos (Casanova, et al., 2018). Este modelo considera el contexto como un aspecto que incide de forma activa en el aprendizaje; por lo cual, el docente parte de las experiencias previas de sus estudiantes, para desarrollar nuevos conocimientos, integrarlos y volverlos significativos; con el fin de aplicarlos en otras situaciones de su vida (Bejar y Quispe, 2020).

Por último, el modelo socioformativo busca una formación integral del estudiante, a través del desarrollo de un proyecto ético de vida y una visión holística; permite la movilización de saberes para solucionar problemas que mejoren la realidad social (Casanova, et al., 2018). Este enfoque, permite una valoración del currículo en forma holística, en la cual se consideran los retos de la sociedad del conocimiento; además, se requiere la atención de los problemas sociales, con la finalidad de favorecer la calidad de vida a través del desarrollo social y económico del contexto inmediato. En este sentido, analizar el contexto interno y externo implica no solo la identificación de problemas del contexto desde la perspectiva particular del área de conocimiento, sino en una perspectiva interdisciplinar (Tobón, 2017).

Otras características de este enfoque son: a) propone la implementación de proyectos formativos como propuesta didáctica preponderante y la figura del docente como mediador y no como catedrático; b) el enfoque en la gestión del talento humano no solo de los estudiantes sino también en los docentes; y, c) promueve la colaboración y participación de los diferentes actores educativos en el proceso de desarrollo curricular, para que al docente se le facilité el desarrollo de dichas competencias durante el proceso educativo. En este sentido, este enfoque permite una gestión curricular con visión global, considerando alianzas, gestionando recursos económicos, materiales y humanos, así como el seguimiento de los resultados de manera periódica; con el fin de desarrollar una formación más integral, articulando los saberes y motivando el compromiso ético de los estudiantes (Tobón, 2017).

Para lograr una gestión curricular holística, la socioformación establece 12 fases para su desarrollo: a) planeación del liderazgo y equipo de trabajo, b) planeación de la realización o mejoras del modelo educativo institucional, c) estudio del contexto interno y externo, d) planeación del perfil de egreso y proceso de egreso, e) planeación del proceso de ingreso por competencias, f) planeación de la malla curricular, g) reglamento de formación y evaluación, h) planeación de la gestión académica, i) planeación de espacios formativos, j) gestión del talento humano por competencias, k) planeación y gestión de recursos, y, I) mediación para la formación integral, el aprendizaje de las competencias y la calidad de vida (Tobón, 2017). Algunos de estas fases, se basan en las metodologías propuestas por otros autores expertos en desarrollo curricular (Casarini, 2013; entre otros). En cada una de estas fases se desarrollan procesos y productos específicos (ver tabla 1).

No obstante, debido a que este enfoque tiene pocos años implementándose, en algunas universidades no se cuenta con información sobre la aplicación de estas 12 fases, como parte de la metodología socioformativa. Por lo anterior, el objetivo del presente estudio fue identificar el grado de aplicación de la gestión curricular holística en el diseño de programas de pregrado en universidades de América Latina, desde la perspectiva de los gestores curriculares y docentes. Además, de comparar el nivel de aplicación de las fases de la gestión curricular holística en las universidades que aplican el modelo por competencias, el enfoque socioformativo y otros modelos curriculares. Por lo cual, esta investigación se realizó considerando universidades latinoamericanas con distintos enfoques curriculares, de tal forma que esta diversidad en los enfoques, permitiera identificar las diferencias en el proceso de desarrollo curricular.

\section{MÉTODO}

Se realizó un estudio exploratorio, con enfoque cuantitativo, alcance descriptivo y comparativo. Es exploratorio, porque se indaga la aplicación de una metodología para el diseño curricular, relativamente nueva: el enfoque Socioformativo. 
Tabla 1. Fases las fases del diseño curricular de acuerdo a la gestión curricular holística

\begin{tabular}{|c|c|c|c|}
\hline & Fases & Características & Productos \\
\hline 1. & $\begin{array}{l}\text { Planeación del } \\
\text { liderazgo y equipo } \\
\text { de trabajo }\end{array}$ & $\begin{array}{l}\text { El equipo se capacita en estrategias de trabajo } \\
\text { colaborativo y busca la articulación de las fortalezas de } \\
\text { todos para lograr con alto impacto las metas propuestas. } \\
\text { Se evalúan los conflictos que se presentan y se buscan } \\
\text { acciones creativas para superarlos, de tal manera que el } \\
\text { trabajo en equipo posea continuidad. }\end{array}$ & $\begin{array}{l}\text { Plan de acción del diseño y } \\
\text { mejoras del plan de } \\
\text { estudios }\end{array}$ \\
\hline 2. & $\begin{array}{l}\text { Planeación de la } \\
\text { realización o } \\
\text { mejoras del modelo } \\
\text { educativo } \\
\text { institucional }\end{array}$ & $\begin{array}{l}\text { El modelo educativo establece acciones creativas para } \\
\text { asegurar su efectiva implementación. Se enfoca en la } \\
\text { innovación curricular para superar el esquema de las } \\
\text { asignaturas o módulos, y promueve la transformación de } \\
\text { las prácticas docentes buscando la transversalidad. } \\
\text { Establece estrategias para el desarrollo del pensamiento } \\
\text { complejo a través de proyectos que se articulan con la } \\
\text { comunidad y las organizaciones. }\end{array}$ & $\begin{array}{l}\text { Documento del modelo } \\
\text { educativo por competencias } \\
\text { bajo el enfoque } \\
\text { socioformativo }\end{array}$ \\
\hline 3. & $\begin{array}{l}\text { Estudio del contexto } \\
\text { interno y externo }\end{array}$ & $\begin{array}{l}\text { El documento de la síntesis del estudio del contexto } \\
\text { considera los problemas del futuro mediante un análisis } \\
\text { prospectivo. Estos problemas tienen la potencialidad de } \\
\text { orientar la formación acorde con las nuevas tendencias } \\
\text { educativas. Los problemas están descritos con claridad, } \\
\text { pueden orientar la formación de los estudiantes e implicar } \\
\text { cambios e innovaciones en el programa. }\end{array}$ & $\begin{array}{l}\text { Documento con el estudio } \\
\text { del contexto interno y } \\
\text { externo }\end{array}$ \\
\hline 4. & $\begin{array}{l}\text { Planeación del perfil } \\
\text { de egreso y } \\
\text { proceso de egreso }\end{array}$ & $\begin{array}{l}\text { El perfil de egreso propone las competencias específicas } \\
\text { y genéricas que deben desarrollar los estudiantes durante } \\
\text { su formación, tomando como base los problemas } \\
\text { actuales y futuros, a nivel local, nacional e internacional. } \\
\text { Se describen acciones concretas para apoyar a los } \\
\text { estudiantes en el logro del perfil de egreso y el proceso } \\
\text { de titulación en tiempo y forma, como también para la } \\
\text { vinculación laboral o la continuidad de los estudios en } \\
\text { otro ciclo. }\end{array}$ & $\begin{array}{l}\text { Documento de perfil de } \\
\text { egreso por competencias y } \\
\text { metodología de acreditación } \\
\text { y seguimiento académico }\end{array}$ \\
\hline 5. & $\begin{array}{l}\text { Planeación del } \\
\text { proceso de ingreso } \\
\text { por competencias }\end{array}$ & $\begin{array}{l}\text { El perfil de ingreso se compone de las competencias } \\
\text { básicas, genéricas y/o específicas esenciales que se } \\
\text { deben poseer al inicio del programa o que se pueden } \\
\text { desarrollar durante los dos primeros años. Se brinda un } \\
\text { programa concreto con módulos opcionales y estrategias } \\
\text { para que los estudiantes fortalezcan las competencias } \\
\text { básicas durante los dos primeros años. }\end{array}$ & $\begin{array}{l}\text { Documento con perfil de } \\
\text { ingreso por competencias, } \\
\text { evaluación, acciones de } \\
\text { reforzamiento y programa } \\
\text { propedéutico }\end{array}$ \\
\hline 6. & $\begin{array}{l}\text { Planeación de la } \\
\text { malla curricular }\end{array}$ & $\begin{array}{l}\text { El mapa curricular está basado en proyectos formativos } \\
\text { que promueven la inter y transdisciplinariedad, con el fin } \\
\text { de resolver los problemas del contexto actual y futuro. El } \\
\text { número de proyectos formativos es de un promedio de } \\
\text { tres por semestre. Tiene ejes creativos y diferenciadores } \\
\text { de otros programas similares en el entorno. Se tienen } \\
\text { acciones para evaluar y mejorar de manera continua el } \\
\text { mapa curricular. }\end{array}$ & $\begin{array}{l}\text { Malla curricular del } \\
\text { programa educativo }\end{array}$ \\
\hline 7. & $\begin{array}{l}\text { Reglamento de } \\
\text { formación y } \\
\text { evaluación }\end{array}$ & $\begin{array}{l}\text { El reglamento establece lineamientos para la formación y } \\
\text { evaluación basados en la inclusión. Se describen } \\
\text { acciones para apoyar a los estudiantes con el fin de que } \\
\text { logren las metas establecidas, tanto al inicio del programa } \\
\text { como en la mitad y al final. }\end{array}$ & Reglamento del estudiante \\
\hline 8. & $\begin{array}{l}\text { Planeación de la } \\
\text { gestión académica }\end{array}$ & $\begin{array}{l}\text { El equipo se capacita en estrategias de trabajo } \\
\text { colaborativo y busca la articulación de las fortalezas de } \\
\text { todos para lograr con alto impacto las metas propuestas. } \\
\text { Se evalúan los conflictos que se presentan y se buscan } \\
\text { acciones creativas para superarlos, de tal manera que el } \\
\text { trabajo en equipo posea continuidad. }\end{array}$ & $\begin{array}{l}\text { Plan de acción del diseño y } \\
\text { mejoras del plan de } \\
\text { estudios }\end{array}$ \\
\hline 9. & $\begin{array}{l}\text { Planeación de } \\
\text { espacios formativos }\end{array}$ & $\begin{array}{l}\text { Se planean proyectos formativos que integran saberes } \\
\text { y promueven la inter y transdisciplinariedad, con énfasis } \\
\text { en el abordaje de problemas de impacto en el entorno. } \\
\text { Se busca tener impacto en el mejoramiento de las } \\
\text { condiciones de vida de un determinado sector de la } \\
\text { sociedad, y a partir de ello se logra la formación } \\
\text { integral. }\end{array}$ & $\begin{array}{l}\text { Proyecto formativos o } \\
\text { integradores }\end{array}$ \\
\hline
\end{tabular}


Tabla 1: continuación.

\begin{tabular}{|c|c|c|c|}
\hline & Fases & Características & Productos \\
\hline 10 & $\begin{array}{l}\text { Gestión del } \\
\text { talento humano } \\
\text { por competencias }\end{array}$ & $\begin{array}{l}\text { Se posee un programa sistémico de gestión del talento } \\
\text { humano, integrado por actividades continuas de desarrollo } \\
\text { del talento en los diversos actores, con el fin de lograr las } \\
\text { metas del currículo. Este programa de gestión del talento } \\
\text { integra estrategias para la selección, formación, evaluación, } \\
\text { promoción y remuneración de todo el personal para lograr } \\
\text { un programa de calidad. }\end{array}$ & $\begin{array}{l}\text { Acciones para la } \\
\text { implementación de la } \\
\text { gestión del talento humano } \\
\text { y proceso de reclutamiento, } \\
\text { selección y formación }\end{array}$ \\
\hline 11 & $\begin{array}{l}\text { Planeación y } \\
\text { gestión de } \\
\text { recursos }\end{array}$ & $\begin{array}{l}\text { Se propone la creación y/o gestión de recursos de } \\
\text { aprendizaje que posibiliten lograr alto impacto en la } \\
\text { formación y en el abordaje de los problemas del contexto, } \\
\text { con base en actividades colaborativas entre diferentes } \\
\text { actores y el apoyo en la investigación pura y aplicada. }\end{array}$ & $\begin{array}{l}\text { Políticas para la gestión de } \\
\text { recursos y su descripción }\end{array}$ \\
\hline 12 & $\begin{array}{l}\text { Mediación para la } \\
\text { formación integral, } \\
\text { el aprendizaje de } \\
\text { las competencias } \\
\text { y la calidad de } \\
\text { vida }\end{array}$ & $\begin{array}{l}\text { En el currículo se describen siete acciones que todos los } \\
\text { docentes deben implementar como mínimo para la } \\
\text { formación de los estudiantes a partir de problemas del } \\
\text { contexto y con flexibilidad: -Visión compartida y motivación } \\
\text { frente al problema y el producto a lograr -Análisis de } \\
\text { saberes previos -Cocreación del conocimiento para } \\
\text { comprender y explicar el problema -Metodología y ejemplo } \\
\text { para planear la resolución del problema -Aplicación } \\
\text { colaborativa para resolver el problema en el contexto - } \\
\text { Mejoramiento del producto -Socialización del producto. En } \\
\text { este nivel considera también la articulación de saberes de } \\
\text { varias asignaturas para abordar los problemas del contexto } \\
\text { con base en la colaboración. }\end{array}$ & $\begin{array}{l}\text { Portafolio de evidencias de } \\
\text { la formación de los } \\
\text { estudiantes }\end{array}$ \\
\hline
\end{tabular}

\section{Participantes}

En este estudio participaron 172 profesionales de la educación, que laboran en 52 universidades de nueve países de América Latina: México, Chile, Perú, Colombia, Guatemala, El Salvador, Costa Rica, Brasil y Uruguay (ver la distribución de los participantes por país en la tabla 2). Los participantes fueron seleccionados por ser integrantes de los equipos de rediseño curricular en sus universidades; dichos equipos conformados por los gestores de los programas educativos y los docentes que colaboran en dichos PE. El $43 \%$ de los participantes fue masculino y el $57 \%$ femenino; en la mayoría de las universidades (66.6\%) participaron, aparte del coordinador del PE de pregrado. Asimismo, el $72 \%$ de los participantes se desempeña en instituciones públicas y el $28 \%$ en privadas; el $55 \%$ gestiona un currículo basado en el modelo por competencias, el $18 \%$ es por competencias, bajo el enfoque socioformativo y el $27 \%$ está basado en otros modelos educativos (ver tabla 3).

Tabla 2. Países de procedencia de los participantes del estudio

\begin{tabular}{|l|c|c|}
\hline País & Frecuencia & Porcentaje \\
\hline México & 124 & $72.1 \%$ \\
\hline Chile & 15 & $8.7 \%$ \\
\hline Perú & 15 & $8.7 \%$ \\
\hline Colombia & 8 & $4.7 \%$ \\
\hline Guatemala & 4 & $2.3 \%$ \\
\hline El Salvador & 3 & $1.7 \%$ \\
\hline Costa Rica & 1 & $0.6 \%$ \\
\hline Brasil & 1 & $0.6 \%$ \\
\hline Uruguay & 1 & $0.6 \%$ \\
\hline
\end{tabular}

Tabla 3. Tipo de modelo curricular de las universidades participantes

\begin{tabular}{|c|c|c|}
\hline Modelo educativo & Frecuencia & Porcentaje \\
\hline Basado en competencias & 95 & $55 \%$ \\
\hline Basado en competencias y bajo el enfoque Socioformativo & 31 & $18 \%$ \\
\hline Otros: Conductista, Humanista y Constructivista & 46 & $27 \%$ \\
\hline
\end{tabular}




\section{Instrumento}

Se utilizó una rúbrica para valorar aplicación de la metodología para el diseño del currículo de PE de pregrado, bajo el enfoque socioformativo. La rúbrica integra doce ítems referentes a cada una de las fases para la gestión curricular holística establecida por Tobón (2017) (ver tabla 4); además de, descriptores que expresan cinco niveles de aplicación de dichas fases, basado en los niveles de desempeño de Hernández et al., (2016): 0) nivel muy bajo, 1) nivel bajo, 2) nivel medio, 3) nivel medio alto, y 4) nivel muy alto (ver tabla 5). Cabe señalar que este instrumento fue sometido previamente a un proceso de validez de contenido, mediante el juicio de doce expertos y ofreció evidencia de su pertinencia y claridad al obtener 0.75 en el índice $\mathrm{V}$ de Aiken. Se probó también la validez de constructo mediante un Análisis Factorial Exploratorio que determinó una estructura factorial de $\mathrm{KMO}$ con un valor de 0.92. Finalmente, se demostró su confiabilidad, debido a que se obtuvo un valor de 0.94 con el Alfa de Cronbach (Crespo et al., 2019).

Tabla 4. Rúbrica para autoevaluar las fases esenciales para la gestión curricular holística

\begin{tabular}{|c|c|c|}
\hline No. & Fases & Ítem \\
\hline 1 & $\begin{array}{l}\text { Planeación del liderazgo y } \\
\text { equipo de trabajo }\end{array}$ & $\begin{array}{l}\text { ¿En qué grado se tiene un equipo líder para gestionar el currículo, con } \\
\text { acciones concretas y periódicas que aseguren su pertinencia? }\end{array}$ \\
\hline 2 & $\begin{array}{l}\text { Planeación de la realización } \\
\text { o mejoras del modelo } \\
\text { educativo institucional }\end{array}$ & $\begin{array}{l}\text { ¿En qué grado se propone un modelo educativo que responde a los retos } \\
\text { de formar para la sociedad del conocimiento, mediante el énfasis en la } \\
\text { resolución de problemas del entorno con pensamiento complejo? }\end{array}$ \\
\hline \multirow[t]{2}{*}{3} & $\begin{array}{l}\text { Planeación del liderazgo y } \\
\text { equipo de trabajo }\end{array}$ & $\begin{array}{l}\text { ¿En qué grado se tiene un equipo líder para gestionar el currículo, con } \\
\text { acciones concretas y periódicas que aseguren su pertinencia? }\end{array}$ \\
\hline & $\begin{array}{l}\text { Planeación de la realización } \\
\text { o mejoras del modelo } \\
\text { educativo institucional }\end{array}$ & $\begin{array}{l}\text { ¿En qué grado se propone un modelo educativo que responde a los retos } \\
\text { de formar para la sociedad del conocimiento, mediante el énfasis en la } \\
\text { resolución de problemas del entorno con pensamiento complejo? }\end{array}$ \\
\hline 4 & $\begin{array}{l}\text { Estudio del contexto interno } \\
\text { y externo }\end{array}$ & $\begin{array}{l}\text { ¿En qué grado el diseño curricular del programa tiene como base un } \\
\text { diagnóstico sistemático y formal de los retos y problemas del contexto tanto } \\
\text { de la actualidad como del futuro, considerando lo local y lo global, con el fin } \\
\text { de orientar la formación, la investigación y la vinculación con la sociedad? }\end{array}$ \\
\hline 5 & $\begin{array}{l}\text { Planeación del perfil de } \\
\text { egreso y proceso de egreso }\end{array}$ & $\begin{array}{l}\text { ¿En qué grado se tiene un perfil de egreso basado en competencias que se } \\
\text { orienta a resolver los problemas y afrontar los retos identificados en el } \\
\text { estudio del contexto? }\end{array}$ \\
\hline 6 & $\begin{array}{l}\text { Planeación del proceso de } \\
\text { ingreso por competencias }\end{array}$ & $\begin{array}{l}\text { ¿En qué grado se posee un perfil de ingreso en el cual se describen con } \\
\text { detalle las competencias básicas y genéricas mínimas que deben poseer } \\
\text { los estudiantes al inicio del programa para lograr con éxito el perfil de } \\
\text { egreso, tener continuidad en los estudios y prevenir la deserción? }\end{array}$ \\
\hline 7 & $\begin{array}{l}\text { Planeación de la malla } \\
\text { curricular }\end{array}$ & $\begin{array}{l}\text { ¿En qué grado la malla curricular del programa se orienta a la multi, inter y } \\
\text { transdisciplinariedad con espacios de formación articulados y en poca } \\
\text { cantidad por semestre, centrados en problemas? }\end{array}$ \\
\hline 8 & $\begin{array}{l}\text { Reglamento de formación y } \\
\text { evaluación }\end{array}$ & $\begin{array}{l}\text { ¿En qué grado se tiene un sistema de evaluación y formación que enfatiza } \\
\text { en la evaluación continua y formativa centrada en el mejoramiento continuo } \\
\text { y el desarrollo del talento a través de productos de aplicación y el empleo de } \\
\text { instrumentos que sean comprendidos por los estudiantes desde el inicio? }\end{array}$ \\
\hline 9 & $\begin{array}{l}\text { Planeación de la gestión } \\
\text { académica }\end{array}$ & $\begin{array}{l}\text { ¿En qué grado se tiene una política concreta de investigación en el } \\
\text { programa mediante líneas de investigación que articulan los espacios } \\
\text { formativos y el trabajo de los docentes y estudiantes, considerando los } \\
\text { problemas del contexto? }\end{array}$ \\
\hline 10 & $\begin{array}{l}\text { Gestión del talento humano } \\
\text { por competencias }\end{array}$ & $\begin{array}{l}\text { ¿En qué grado se propone un cuerpo directivo, docente y administrativo con } \\
\text { la descripción de las competencias necesarias para poner en marcha el } \\
\text { currículo, junto con un proceso de formación continua y la evaluación } \\
\text { periódica del desempeño, que lleven a que el programa tenga impacto en la } \\
\text { formación de las personas de acuerdo con la sociedad del conocimiento? }\end{array}$ \\
\hline 11 & $\begin{array}{l}\text { Planeación de espacios } \\
\text { formativos }\end{array}$ & $\begin{array}{l}\text { ¿En qué grado se presenta el microcurrículo centrado en la resolución de } \\
\text { problemas del entorno, la evaluación por medio de productos e } \\
\text { instrumentos, y el desarrollo de competencias genéricas para la sociedad } \\
\text { del conocimiento, tales como el pensamiento crítico, la sustentabilidad } \\
\text { ambiental, la colaboración, el emprendimiento, el inglés, la comunicación } \\
\text { escrita, la gestión del conocimiento, etc.? }\end{array}$ \\
\hline 12 & $\begin{array}{l}\text { Planeación y gestión de } \\
\text { recursos }\end{array}$ & $\begin{array}{l}\text { ¿En qué grado se proponen recursos que ayuden a formar para la sociedad } \\
\text { del conocimiento, como laboratorios o diversos ambientes de aprendizaje } \\
\text { que permitan resolver problemas del entorno o las tecnologías de la } \\
\text { información y la comunicación que posibiliten comunidades de aprendizaje } \\
\text { para el desarrollo social? }\end{array}$ \\
\hline
\end{tabular}




\section{Procedimiento}

Para llevar a cabo esta investigación se desarrollaron las siguientes fases: 1) Selección de la muestra: se creó una base de datos con las universidades a quienes se invitó a participar en la investigación, tomando como referencia las redes de colaboración de la universidad (ITSON), donde se incluyó a las instituciones con las que se tiene intercambio de movilidad académica, identificándose a los responsables de los PE. Así mismo, se solicitó apoyo a profesores adscritos a instituciones que colaboran con el instituto CIFE. 2) Digitalización y aplicación del instrumento: Se integró el instrumento en un formulario de Google, con la finalidad de enviar la liga para que los participantes pudieran acceder al mismo. 3) Aplicación del instrumento: Se enviaron las invitaciones a los participantes seleccionados, mediante un correo electrónico, donde se explicó el objetivo del estudio, la liga para acceder al instrumento y al consentimiento informado; además, se dio seguimiento a la aplicación con la finalidad de asegurar la obtención de los datos requeridos. 4) Análisis de los resultados: se integró una base de datos con los resultados de las respuestas de los participantes y se procesaron con el paquete estadístico SPSS ver. 24. Se realizó la prueba Kruskal-Wallis para comparar las medias de las fases del proceso de diseño curricular entre los modelos que utilizan los participantes de diferentes universidades e identificar si existía diferencia significativa entre los tres tipos de modelos identificados. Se seleccionó esta prueba, análoga a la prueba paramétrica ANOVA, debido a que puede efectuarse en mediciones ordinales al comparar más dos muestras independientes y en instrumentos cuyas condiciones no permiten la parametricidad de sus variables, en este caso una rúbrica (Ostertagová et al., 2013).

Tabla 5. Niveles de aplicación de las fases esenciales para la gestión curricular holística de Hernández et al., (2016).

\begin{tabular}{|l|l|}
\hline \multicolumn{1}{|c|}{ Nivel } & \multicolumn{1}{c|}{ Descriptor } \\
\hline $\begin{array}{l}\text { 0) nivel muy } \\
\text { bajo }\end{array}$ & $\begin{array}{l}\text { Se tienen algunos elementos en el área, pero no están relacionados con el desempeño esperado } \\
\text { en la fase de diseño curricular }\end{array}$ \\
\hline 1) nivel bajo & $\begin{array}{l}\text { Se tiene recepción de información, el desempeño es operativo y se tienen nociones sobre la } \\
\text { realidad y en el ámbito de actuación de la fase de diseño curricular }\end{array}$ \\
\hline 2) nivel medio & $\begin{array}{l}\text { Se resuelven problemas sencillos del contexto, hay labores de asistencia a otras personas, se } \\
\text { tienen elementos técnicos de los procesos implicados y se poseen algunos conceptos básicos de } \\
\text { la fase de diseño curricular }\end{array}$ \\
\hline $\begin{array}{l}\text { 3) nivel medio } \\
\text { alto }\end{array}$ & $\begin{array}{l}\text { No se requiere de asesoría continua de otras personas, se gestionan recursos, hay argumentación } \\
\text { sólida y profunda, se resuelven problemas de diversa índole con los elementos necesarios para el } \\
\text { diseño curricular }\end{array}$ \\
\hline $\begin{array}{l}\text { 4) nivel muy } \\
\text { alto }\end{array}$ & $\begin{array}{l}\text { Se plantean estrategias de cambio en la realidad, hay creatividad e innovación, se muestran altos } \\
\text { niveles de impacto en la realidad, se hacen análisis evolutivos y prospectivos para abordar mejor } \\
\text { los problemas, y se consideran las consecuencias de diferentes opciones de resolución de los } \\
\text { problemas en el contexto al realizar el diseño curricular }\end{array}$ \\
\hline
\end{tabular}

\section{RESULTADOS Y DISCUSIÓN}

Se llevó a cabo el análisis de las medias de las 12 fases esenciales para la gestión curricular holística, obteniendo una media de 2.4, con una desviación estándar de 1.02; es decir, los participantes del estudio consideran que su PE aplica las fases para la gestión curricular en un nivel medio. La fase cuatro relacionada con la planeación del perfil de egreso y proceso de egreso se ubicó con la media más alta de 2.94, mientras que la fase siete del reglamento de formación y evaluación obtuvo la más baja de 2.16. Asimismo, las universidades donde se desarrollan procesos de diseño curricular bajo otros modelos: conductista o humanista, el nivel de aplicación de las fases curriculares se ubican en un nivel bajo y medio. No obstante, los PE que se diseñaron bajo el enfoque por competencias y socioformativo se ubican en un nivel medio o medio alto (ver tabla 6). Estos resultados coinciden con lo establecido por Casarini (2013) en cuanto a las ventajas del enfoque por competencias; además de Icarte y Labate (2016) y Parra et al. (2017) quienes concluyen que con una metodología bajo este enfoque, se desarrolla un proceso más ordenado y sistemático, facilita la toma de decisiones y el involucramiento de docentes, lo cual impacta positivamente en la efectividad del proceso formativo y el logro del perfil de egreso.

Los valores más altos se ubicaron en la fase cuatro (ver tabla 6) planeación del perfil y proceso de egreso, existiendo coincidencia como media más alta en los tres modelos analizados, es decir, el proceso de egreso se planea de forma puntual para asegurar que durante el proceso formativo los estudiantes alcancen las competencias establecidas en el plan de estudios, a fin de que puedan desarrollarse pertinentemente en el contexto laboral. Lo anterior, indica que esta es una de las principales fases del proceso de diseño curricular independientemente del modelo utilizado, no obstante, los valores más altos se obtuvieron en los enfoques por competencias y socioformativo. Esta fase es primordial, debido a que se definen las competencias, propósitos u objetivos del perfil de egreso, con base a los problemas del contexto social y profesional; así como, especificar las acciones que se requieren para el logro de dichos propósitos y definir las estrategias del proceso formativo (Casarini, 2013; Icarte y Labate, 2016; Rowe y Zegwaard, 2017; Tobón, 2017). 
Tabla 6. Nivel de aplicación de las fases para la gestión curricular holística en PE con diferentes modelos educativos

\begin{tabular}{|c|c|c|c|c|c|c|c|c|c|}
\hline \multirow{2}{*}{$\begin{array}{c}\text { Fases del } \\
\text { currículo }\end{array}$} & \multicolumn{3}{|c|}{ Modelo Socioformativo } & \multicolumn{3}{|c|}{ Modelo por Competencias } & \multicolumn{3}{c|}{ Otros Modelos } \\
\cline { 2 - 10 } & $M$ & $D S$ & Nivel & $M$ & $D S$ & Nivel & $M$ & $D S$ & Nivel \\
\hline 1 & 2.71 & .93 & Medio & 2.31 & 1.26 & Medio & 1.87 & 1.60 & Medio \\
\hline 2 & 2.77 & .95 & Medio & 2.71 & 1.15 & Medio & 2.15 & 1.50 & Medio \\
\hline 3 & 2.84 & 1.03 & Medio & 2.72 & 1.028 & Medio & 2.09 & 1.44 & Medio \\
\hline 4 & 3.13 & .88 & Medio alto & 3.09 & .99 & Medio alto & 2.50 & 1.54 & Medio alto \\
\hline 5 & 2.74 & 1.15 & Medio & 2.64 & 1.07 & Medio & 1.96 & 1.47 & Bajo \\
\hline 6 & 2.94 & .96 & Medio & 2.56 & .99 & Medio & 2.00 & 1.30 & Medio \\
\hline 7 & 2.39 & 1.14 & Medio & 2.28 & 1.05 & Medio & 1.76 & 1.41 & Bajo \\
\hline 8 & 2.52 & 1.06 & Medio & 2.38 & 1.27 & Medio & 1.87 & 1.51 & Bajo \\
\hline 9 & 2.68 & .97 & Medio & 2.40 & 1.14 & Medio & 2.00 & 1.39 & Medio \\
\hline 10 & 2.65 & 1.19 & Medio & 2.33 & 1.10 & Medio & 1.91 & 1.38 & Bajo \\
\hline 11 & 2.55 & 1.15 & Medio & 2.55 & 1.13 & Medio & 2.00 & 1.53 & Medio \\
\hline 12 & 2.48 & 1.06 & Medio & 2.31 & 1.03 & Medio & 1.96 & 1.39 & Bajo \\
\hline
\end{tabular}

Por el contrario, las medias más bajas fueron coincidentes en la fase siete (ver tabla 6) que corresponde al reglamento de formación y evaluación; lo cual indica que los participantes de este estudio, independientemente de la universidad y modelo curricular utilizado, efectúan en menor medida las actividades de esta fase. Aclarando que se obtuvo un nivel bajo en los enfoques diferentes al de competencias y socioformativo donde el nivel fue medio. Esto puede deberse a que en las universidades con otros modelos curriculares, a diferencia de lo establecido por varios expertos en el tema (Shubert, 2018; Tobón, 2017), no le otorgan mucha importancia a esta fase que consiste en instituir lineamientos para evaluar el progreso del estudiante durante todo el proceso formativo, con el fin de identificar en qué medida se están alcanzando las competencias u objetivos del perfil de egreso, de donde se derivarán las decisiones para desarrollar estrategias de mejora.

Adicionalmente, se realizó una comparación de medias, a partir de la prueba Kruskal-Wallis, con la finalidad de identificar si existen diferencias significativas entre las puntuaciones establecidas por los participantes en las fases de diseño curricular y el modelo curricular en el que se basaron para el desarrollo de los planes de estudio de los PE. Las fases en las cuales no se identificó una diferencia significativa fueron: 1) Planeación del liderazgo y del equipo de trabajo, 2) Planeación de la realización o mejoras del modelo educativo institucional, 4) Planeación del perfil de egreso y proceso de egreso, 8) Planeación de la gestión académica, 9) Planeación de espacios formativos, 11) Planeación y gestión de recursos; y, 12) Mediación para la formación integral, el aprendizaje de las competencias y la calidad de vida (ver tabla 7).

Tabla 7. Diferencias entre universidades en la aplicación del enfoque por competencias y otros enfoques en su currículo * $p<.05$. ${ }^{\star *} p<.01 .{ }^{* * *} p<.001$

\begin{tabular}{|c|c|c|c|c|c|c|c|c|}
\hline \multirow{2}{*}{$\begin{array}{c}12 \text { fases } \\
\text { esenciales } \\
\text { del currículo }\end{array}$} & \multicolumn{2}{|c|}{ Modelo socioformativo } & \multicolumn{2}{|c|}{ Modelo por competencias } & \multicolumn{2}{|c|}{ Otros modelos } & \multirow{2}{*}{$\begin{array}{c}87 \\
h \\
\text { (valor de } \\
\text { la prueba) }\end{array}$} & \multirow[b]{2}{*}{$p$} \\
\hline & $M$ & $D E$ & $M$ & $D E$ & $M$ & $D E$ & & \\
\hline 1 & 2.71 & .93 & 2.31 & 1.26 & 1.87 & 1.60 & 4.45 & .107 \\
\hline 2 & 2.77 & .95 & 2.71 & 1.15 & 2.15 & 1.50 & 10.18 & 122 \\
\hline 3 & 2.84 & 1.03 & 2.72 & 1.028 & 2.09 & 1.44 & 7.12 & $.029^{\star \star}$ \\
\hline 4 & 3.13 & .88 & 3.09 & .99 & 2.50 & 1.54 & 13.22 & 176 \\
\hline 5 & 2.74 & 1.15 & 2.64 & 1.07 & 1.96 & 1.47 & 9.51 & $.021^{\star \star}$ \\
\hline 6 & 2.94 & .96 & 2.56 & .99 & 2.00 & 1.30 & 10.10 & $.003^{\star \star \star}$ \\
\hline 7 & 2.39 & 1.14 & 2.28 & 1.05 & 1.76 & 1.41 & 4.89 & $.034^{\star \star}$ \\
\hline 8 & 2.52 & 1.06 & 2.38 & 1.27 & 1.87 & 1.51 & 2.36 & .114 \\
\hline 9 & 2.68 & .97 & 2.40 & 1.14 & 2.00 & 1.39 & 6.59 & .100 \\
\hline 10 & 2.65 & 1.19 & 2.33 & 1.10 & 1.91 & 1.38 & 7.48 & $.048^{*}$ \\
\hline 11 & 2.55 & 1.15 & 2.55 & 1.13 & 2.00 & 1.53 & 5.10 & .132 \\
\hline 12 & 2.48 & 1.06 & 2.31 & 1.03 & 1.96 & 1.39 & 1.54 & .269 \\
\hline
\end{tabular}


Lo anterior significa que el modelo curricular que se utiliza en las universidades participantes, no hace diferencia alguna en estas fases del proceso de diseño curricular. Estos resultados se asemejan a los hallazgos de Changiz et al. (2019) y Ngassapa et al. (2012) quiénes concluyeron que para desarrollar procesos curriculares se requiere una directiva que lidere el cambio y tome las decisiones pertinentes. Asimismo, coinciden con los resultados de Aguirre-Garzón (2018), Cisternas, et al. (2016), García-Martínez et al., (2018); quienes resaltan la importancia de la participación de los profesores y la creación de un comité o equipo académico interdisciplinario que colabore para el éxito de este proceso.

Específicamente, en la fase de planeación y gestión de recursos, este hallazgo es similar a los obtenidos por Alexander y Hjortsø (2019) y Changiz et al. (2019) quienes identificaron que en los procesos de diseño curricular se requiere movilización recursos, además de contar con principios claros que regulen las fases del desarrollo curricular, con el fin de favorecer una amplia comunicación en el proceso para alcanzar los principios que regirán el currículo. Esta fase es de suma importancia debido a que es el punto de partida en los procesos de diseño curricular en las instituciones de educación superior (Icarte y Labate, 2016; Ngassapa et al., 2012). Por otra parte, sí se identificó una diferencia significativa en las siguientes fases: 3) Estudio del contexto interno y externo, el cual es una base para el proceso de actualización del currículo; 5) Planeación del proceso de ingreso por competencias, 6) Planeación de la malla curricular; 7) Reglamento de formación y evaluación; y 10) Gestión del talento humano por competencias. Es decir, los programas que se desarrollaron bajo el enfoque socioformativo, cuentan con una media más alta en comparación con los que se basan en otros modelos curriculares, lo cual indica que les otorgan mayor importancia a los elementos de estas 5 fases (ver tabla 7).

Con respecto al estudio del contexto interno y externo, este hallazgo coincide con los resultados de los estudios de Barzaga et al. (2019) y Salas (2016); quienes indican que para que un currículo sea pertinente, debe ser acorde a las necesidades sociales. Es decir, es fundamental que en el plan de estudios se aborden problemas del contexto para que los estudiantes desarrollen habilidades complejas, que las pongan en práctica para identificar y resolver problemas reales, con el fin de darle sentido a su educación y pertinencia al plan de estudios (Barzaga et al., 2019; Glatthorn et. al., 2019; 2013; Rowe y Zegwaard, 2017).

Referente a las fases de planeación del proceso de ingreso por competencias y planeación de la malla curricular, este hallazgo coincide con los resultados de varios estudios (Cisternas et al., 2016; Glatthorn et al., 2019; Icarte y Labate, 2016) donde explican la importancia de estas fases en el proceso de diseño curricular. Es decir, estos estudios enfatizan la trascendencia del plan de estudios o malla curricular, debido a que su estructura permite visualizar la ruta de aprendizaje (Glatthorn et. al., 2019; Icarte y Labate, 2016), igualmente indican que es necesario establecer los aprendizajes esperados y procesos requeridos en el proceso formativo.

Además de la planificación de la experiencia de aprendizaje, es muy importante el establecimiento de criterios de evaluación, los cuáles son cruciales para la fase siete (Reglamento de formación y evaluación), debido a que para asegurar el logro del aprendizaje esperado, se debe evaluar a los estudiantes al inicio, en la mitad y al final del plan de estudios. Lo cual coincide con los hallazgos de Icarte y Labate (2016), López et al. (2016), y Shubert (2018) quienes incluyeron esta fase en sus procesos de rediseño curricular con el fin de diseñar los mecanismos de evaluación de competencias o instituyeron una estrategia de mapeo curricular para la definición de la práctica docente y de evaluación (Icarte y Labate, 2016). En cuanto a la fase de gestión del talento humano, este hallazgo coincide con lo obtenido por Dempster, et al. (2012), Huang (2017), Martínez et al., (2017), quienes resaltan la importancia de la capacitación de profesores y demás actores educativos involucrados en procesos de diseño curricular.

Así mismo, otra de las ventajas de los programas educativos basado en el modelo por competencias y el enfoque socioformativo, a diferencias de los otros enfoques, es que aplican en mayor medida las siguientes fases de la gestión curricular holística: 3) Estudio del contexto interno y externo, el cual es una base para el proceso de actualización del currículo; 5) Planeación del proceso de ingreso por competencias, que permite asegurar que el estudiante cuente con las bases conceptuales y procedimentales, las cuáles puede fortalecer durante los dos primeros años de estudios, porque las requiere para el desarrollo de las competencias del perfil de egreso; y, 6) Planeación de la malla curricular en función de saberes propios para dar solución a los problemas del contexto a través de proyectos formativos, lo que permitirá al estudiante aplicar sus competencias y ser evaluado a través de criterios basados en normas de competencias e instrumentos validados.

Por otra parte, los resultados de esta investigación pueden tener la siguiente utilidad: 1) permitir que los coordinadores de los PE realicen procesos de evaluación curricular con base en los criterios propuestos por la gestión curricular holística; 2) favorecer la toma de decisiones a partir de los resultados obtenidos con la metodología holística, con la finalidad de que se determine que enfoque curricular es el más pertinente para el diseño de sus programas educativos; 3 ) gestionar el cambio del enfoque curricular con los actores que 
participan en el proceso educativo, considerando metodologías como la de Lewin, donde se trabaja con la de los individuos a través de un proceso de descongelamiento, de desplazamiento o avance y por último una fase de recongelamiento (Hornstein, 2015); y c) capacitar al personal docente en los principios, estrategias y criterios que fundamentan el enfoque socioformativo, tales como: emprendimiento, proyecto ético de vida, metacognición, trabajo colaborativo, gestión del conocimiento, evaluación de competencias socioformativas, entre otras.

\section{CONCLUSIONES}

De acuerdo a los resultados obtenidos en esta investigación y su comparación con los hallazgos de otros autores, se derivan las siguientes conclusiones: a) los programas educativos diseñados bajo el enfoque por competencias y socioformativo, cumplen en mayor medida las fases esenciales para la gestión curricular holística; b) la fase de planeación del perfil y proceso de egreso, es primordial debido a que en ésta se definen las competencias del perfil de egreso y deben cubrir las necesidades sociales donde se desempeñará el futuro profesionista; y, c) el proceso de gestión curricular basado en el modelo por competencias con enfoque socioformativo contribuye bastante al logro de esta fase, debido que inicia con un análisis de problemas del contexto y propicia el desarrollo de estrategias que permiten al estudiante alcanzar las competencias de su perfil de egreso desde el inicio del plan de estudios.

\section{AGRADECIMIENTOS}

Esta investigación se realizó con el apoyo del Programa de Fortalecimiento para la Investigación PROFAPI2020-033: Docencia formativa en el programa educativo de LCE, del Instituto Tecnológico de Sonora.

\section{REFERENCIAS}

Aguirre-Garzón, E. A. Unlicensed EFL teachers co-constructing knowledge and transforming curriculum through collaborative-reflective inquiry. http://dx.doi.org/10.15446/profile.v20n1.62323. Profile issues in teachers' professional development, 20 (1), 73-87 (2018).

Alexander, I.K., y Hjortsø, C.N. Sources of complexity in participatory curriculum development: an activity system and stakeholder analysis approach to the analyses of tensions and contradictions. https://doi.org/10.1007/s10734-018-0274x. High educ, 77, 301-322 (2019).

Barzaga, O., Santos, C., Bolívar, O., y Zambrano, J. Gestión del diseño curricular universitario en Ecuador. http://dx.doi.org/10.37960/revista.v24i88.30178. Revista Venezolana de Gerencia, 24 (88), 1262-1271 (2019).

Bejar, L. H., y Quispe, F. D. Educación constructivista: un compromiso transformador. https://doi.org/10.30827/publicaciones.v50i2.13944. Publicaciones, 50(2), 73-85 (2020).

Boyle, B., y Charles, M. Curriculum development: a guide for educators. https://tinyurl.com/y3pbsk5k. C\&M Digitals. Chennai, India (2016).

Casanova, I., Canquiz, L., Paredes, Í., y Inciarte, A. Visión general del enfoque por competencias en Latinoamérica. http://hdl.handle.net/11323/5282, ISSN 2477-9431. Revista de Ciencias Sociales (RCS), 24(4), 114-125 (2018).

Changiz, T., Yamani, N., y otros tres autores. Curriculum management/monitoring in undergraduate medical education: a systematized review. https://doi.org/10.1186/s12909-019-1495-0. BMC Medical Education, 19(60), 1-9 (2019).

Casarini Ratto, M. Teoría y Diseño Curricular (3 $3^{\text {era }}$ ed.). ITESM y Trillas, Ciudad de México, México (2013).

Cisternas, M., Rivera, S., y otros cinco autores. Reforma curricular de la carrera de Medicina de la Pontificia Universidad Católica de Chile. https://dx.doi.org/10.4067/S0034-98872016000100013. Revista médica de Chile, 144 (1), 102-107 (2016).

Crespo, A., Mortis, S., Herrera, S., y Manig, A. Diseño de una rúbrica para autoevaluar los ejes esenciales del currículo de licenciatura desde la socioformación. ISSN 1682-2749. Revista Digital Atenas, 3 (47), 142-160 (2019).

Dempster, J., Benfield, G., y Francis R. An academic development model for fostering innovation and sharing in curriculum design. DOI https://doi.org/10.1080/14703297.2012.677595. Journal Innovations in Education and Teaching International, 49(2),135-147. (2012).

Díaz, V., Poblete, Á., y Gallardo, M. Rediseño curricular por competencias: experiencia en la formación inicial universitaria en Chile. https://doi.org/10.22201/iisue.20072872e.2019.27.341_.Revista iberoamericana de educación superior, 10(27), 72-91 (2019).

García-Martínez, J. A., Cerdas-Montano, V., y Torres-Vitoria, N. Gestión curricular en centros educativos costarricenses: Un análisis desde la percepción docente y la dirección. ttps://doi.org/10.15359/ree.22-1.11. Revista Electrónica Educare, 22 (1), 225-252 (2018).

Glatthorn, A., Boschee, F., Whitehead, B., y Boschee, B. Curriculum leadership. Strategies for development and implementation. Fifth edition. SAGE publications, Los Angeles (2019). 
Hernández, J. Tobón, S., y Guerrero, G. Hacia una evaluación integral del desempeño: las rúbricas socioformativas. ISSN 1665-0441. Revista Ra Ximhai, 12 (6), 359-376 (2016).

Hornstein, $\mathrm{H}$. The integration of project management and organizational change management is now a necessity. https://doi.org/10.1016/j.jproman.2014.08.005. International Journal of Project Management, 33(2), 291 -298 (2015).

Huang, F. The impact of mass and universal higher education on curriculum and instruction: case studies of China and Japan. https://doi.org/10.1007/s10734-016-0061-5. High Educ, 74, 507-525 (2017).

Icarte, G. A., y Labate, H. A. Metodología para la revisión y actualización de un diseño curricular de una carrera universitaria incorporando conceptos de aprendizaje basado en competencias. http://dx.doi.org/10.4067/S071850062016000200002. Formación universitaria, 9 (2), 03-16 (2016).

López, C., Benedito, V., y León, M. (2016). El Enfoque de competencias en la formación universitaria y su impacto en la evaluación: la perspectiva de un grupo de profesionales expertos en pedagogía. https://dx.doi.org/10.4067/S071850062016000400003. Formación universitaria, 9 (4), 11-22 (2016).

Martínez, J., Tobón, S., y Romero, A. Problemáticas relacionadas con la acreditación de la calidad de la educación superior en América Latina. ISNN 1665-2673. Revista de innovación educativa, 17 (73), 79-96 (2017).

Ngassapa, O., Kaaya, E., y otros 12 autores. Curricular transformation of health professions education in Tanzania: The process at Muhimbili University of Health and Allied Sciences (2008-2011). https://doiorg.itson.idm.oclc.org/10.1057/jphp.2012.43. J Public Health Pol, 33, 64-91 (2012).

Ostertagová, E., Ostertag, O., y Kováč, J. Methodology and Application of the Kruskal-Wallis Test. https://doi.org/10.4028/www.scientific.net/amm.611.115. Applied Mechanics and Materials, 611, 115-120 (2014).

Parra, H., Tobon, S., y otros 10 autores. Teachers recognize the need to develop their competencies to improve medical students' performance. International journal of environmental and science education, 12 (8), 1895-1911 (2017).

Pellón, R. Watson, Skinner y algunas disputas dentro del conductismo. ISSN 0121-5469. Revista Colombiana de Psicología, 22(2), 389-399 (2013).

Posso, R. J., Barba, L. C., y Otáñez, N. R. El conductismo en la formación de los estudiantes universitarios. https://doi.org/10.46498/reduipb.v24i1.1229. Revista EDUCARE - UPEL-IPB - Segunda Nueva Etapa 2.0, 24(1), 117-133(2020).

Rowe, A., y Zegwaard, K. Developing graduate employability skills and attributes: curriculum enhancement through work-integrated learning. Asia-Pacific Journal of Cooperative Education, 18, 2, 87-99 (2017).

Salas, R. S. ¿El rediseño curricular sin evaluación curricular es científico?. ISSN 1561-2902. Educación Médica Superior, 30 (2) (2016).

Shubert, W. Perspectives on evaluation from curricular context. https://doi.org/10.14507/epaa.26.3812. Education policy analysis- archives, 26 (47) (2018).

Tobón, S. Essential axes of knowledge society and socioformation. dx.doi.org/10.24944/isbm.978-1-945721-199Education. Kresearch, Mount Dora, U.S.A (2017).

Voogt, M., Pieters, J. M., y Handelzalts, A. Teacher collaboration in curriculum design teams: effects, mechanisms, and conditions. https://doi.org/10.1080/13803611.2016.1247725. Educational Research and Evaluation, 22, 121-140 (2016). 
